\title{
Electroluminescence enhancement in mid-infrared InAsSb resonant cavity light emitting diodes for $\mathrm{CO}_{2}$ detection
}

\author{
Furat A. Al-Saymari ${ }^{1,2, a)}$, Adam P. Craig ${ }^{1}$, Yasir J. Noori ${ }^{3}$, Qi Lu ${ }^{1}$, Andrew R. J. Marshall ${ }^{1}$ \\ and Anthony Krier ${ }^{1, a)}$ \\ ${ }^{1}$ Physics Department, Lancaster University, Lancaster, LA1 4YB, United Kingdom \\ ${ }^{2}$ Department of Physics, College of Education for Pure Science, Basra University, Basra, 61004, Iraq \\ ${ }^{3}$ Electronics and Computer Science Department, University of Southampton, University Road, Southampton, \\ SO17 1BJ, United Kingdom \\ a) Corresponding Author: a.krier@lancaster.ac.uk
}

\begin{abstract}
:
In this work we demonstrated a mid-infrared resonant cavity light emitting diode (RCLED) operating near $4.2 \mu \mathrm{m}$ at room temperature, grown lattice-matched on a GaSb substrate by molecular beam epitaxy, suitable for $\mathrm{CO}_{2}$ gas detection. The device consists of a $1 \lambda$-thick micro-cavity containing an $\operatorname{InAs} \mathrm{s}_{0.90} \mathrm{Sb}_{0.1}$ active region sandwiched between two high contrast, lattice-matched $\mathrm{AlAs}_{0.08} \mathrm{Sb}_{0.92} / \mathrm{GaSb}$ distributed Bragg reflector (DBR) mirrors. The electroluminescence emission spectra of the RCLED were measured over the temperature range from 20 to $300 \mathrm{~K}$ and compared with a reference LED without DBR mirrors. The RCLED exhibits a strong emission enhancement due to resonant cavity effects. At room temperature, the peak emission and the integrated peak emission were found to be increased by a factor of $\sim 70$ and $\sim 11$, respectively, while the total integrated emission enhancement was $\sim \mathrm{x} 33$. This is the highest resonant cavity enhancement ever reported for a mid-infrared LED operating at this wavelength. Furthermore, the RCLED also exhibits superior temperature stability $\sim 0.35 \mathrm{~nm} / \mathrm{K}$ and a significantly narrower (10x) spectral linewidth. High spectral brightness and temperature stable emission entirely within the fundamental absorption band are attractive characteristics for the development of next generation $\mathrm{CO} 2$ gas sensor instrumentation.
\end{abstract}


Greenhouse gases such as $\mathrm{CH}_{4}, \mathrm{CO}_{2}$ and $\mathrm{CO}$ have strong fingerprint absorptions in the midinfrared 3-5 $\mu \mathrm{m}$ spectral region, enabling gas specific detection and remote sensing. For many applications, mid-infrared LEDs are a far more attractive, cost-effective alternative to lasers, especially for widespread distributed sensing applications requiring many point sensors ${ }^{1-8}$. Various LED structures have been developed for $\mathrm{CO}_{2}$ monitoring at $4.2 \mu \mathrm{m}{ }^{9-12}$, including bulk heterostructures of $\operatorname{InAsSbP/InAsSb}{ }^{13}, \mathrm{AlInSb}^{14}, \mathrm{InAsSb}^{15}$, as well as InSb/InAs quantum $\operatorname{dots}^{16}$, InAs/InAsSb quantum wells and superlattices ${ }^{3,5,17,18}$. These devices typically exhibit $300 \mathrm{~K}$ output powers of a few microwatts, with an emittance of $\sim 1-14 \mathrm{~mW} / \mathrm{cm}^{-2}$, but with broadband emission spectra resulting in low available power at the target wavelength. Furthermore, optical extraction efficiency is limited to a few per cent by the large refractive index mismatch between the semiconductor and the air. ${ }^{19,20}$ One solution to this problem involves the use of a resonant cavity to enhance the light emission. This has been achieved at shorter wavelengths by locating the active region inside a Fabry-Perot micro-cavity consisting of two distributed Bragg reflectors (DBRs) to form a resonant cavity light emitting diode (RCLED) structure. RCLEDs offer many advantages including; higher brightness than their conventional LED counterparts, improved directionality, increased spectral purity and power efficiency. $^{21,22}$ Consequently, some research effort has been devoted towards developing RCLEDs and vertical cavity surface emitting laser (VCSELs) for longer wavelength mid-IR applications. $^{23-29}$ There has been a demonstration of a promising mid-IR VCSEL emitting at $4.0 \mu \mathrm{m}$ with single-mode $\mathrm{cw}$ operation up to $-7^{\circ} \mathrm{C} .{ }^{29}$ However, there are only a limited number of reports of RCLEDs that emit at a wavelength of $\sim 4.2 \mu \mathrm{m}$ and these have shown a relatively low resonant cavity emission enhancement. ${ }^{24,30}$

In this work, we present a mid-infrared RCLED tailored for $\mathrm{CO}_{2}$ detection at $4.2 \mu \mathrm{m}$ using high contrast $\mathrm{AlAsSb} / \mathrm{GaSb} \mathrm{DBR}$ mirrors and $\mathrm{InAsSb}$ as the active region. We report the results of numerical modelling to optimize the design of the structure, followed by systematic 
electroluminescence spectroscopy measurements of our RCLED and an InAsSb bulk reference LED at different temperatures to demonstrate the enhancement achieved as a result of using a resonant cavity.

The structure of the RCLED is illustrated in Fig. 1(a) and consists of a $1 \lambda$-thick cavity sandwiched between two distributed Bragg reflectors (DBRs). The active region (bulk InAs $\left.{ }_{0.90} \mathrm{Sb}_{0.10}\right)$ is placed at the antinode of the electric field intensity in order to obtain maximum enhancement. Growth was carried out using a Veeco GenXplor molecular beam epitaxy (MBE) reactor with SUMO cells for Al, Ga and In, and valved cracker cells for As and $\mathrm{Sb}$ fluxes respectively. The first grown layer was an n-type $\left(1 \times 10^{17} \mathrm{~cm}^{-3}\right) \mathrm{GaSb}$ buffer layer on (001) $n$-doped GaSb substrate, followed by a 13.5 pairs lattice-matched un-doped $\mathrm{AlAs} 0.08$ $\mathrm{Sb}_{0.92} / \mathrm{GaSb}$ bottom-DBR mirror, grown at a substrate temperature of $T_{g r}=505{ }^{\circ} \mathrm{C}$. On the top of the bottom-DBR, a $1 \lambda$-thick micro-cavity was grown as follows: an $n$-doped $\operatorname{InAs}_{0.90} \mathrm{Sb}_{0.10}$ layer; $i$-InAs ${ }_{0.90} \mathrm{Sb}_{0.1} ; p$-InAs $0.90 \mathrm{Sb}_{0.10} ;$ a 56 nm-thick $p$-doped $\mathrm{AlAs}_{0.08} \mathrm{Sb}_{0.92}$ barrier layer; and a $p$-doped InAs $0.90 \mathrm{Sb}_{0.10}$ layer. The growth temperature of the cavity layers was fixed at $450{ }^{\circ} \mathrm{C}$, except for the barrier layer which was grown at $530{ }^{\circ} \mathrm{C}$. The top-DBR mirror comprising 5 pairs of $p$-doped $\mathrm{AlAs}_{0.08} \mathrm{Sb}_{0.92} / \mathrm{GaSb}$ was grown at $505{ }^{\circ} \mathrm{C}$. The $n$-type and $p$-type dopants were Te and $\mathrm{Be}$, respectively. The total thickness of the completed RCLED structure is around $13 \mu \mathrm{m}$. A scanning electron microscope (SEM) image of the grown structure in cross-section is shown in Fig. 1(b).

Processing was carried out using standard photolithography and wet chemical etching followed by $\mathrm{Ti} / \mathrm{Au}$ metallization for the ohmic contacts. In particular, InAsSb layers were etched using citric acid: $\mathrm{H}_{2} \mathrm{O}_{2}(2: 1)$. GaSb and $\mathrm{AlAsSb}$ were etched using a dilute ammonia based etchant. A $250 \mathrm{~nm}$ thick $p$-type top metal contact of Ti/Au was deposited on the top $p$ type DBR layer by thermal evaporation and metal lift-off. A second photolithography step was carried out to pattern the sample for subsequent wet etching of the mesa, by etching through 
the structure and stopping within the $n$-InAsSb layer. Finally, a Ti/Au metal contact was then deposited on the $n$-InAsSb layer to provide an ohmic contact for the n-side (see Fig. 1(c)). After fabrication, optical transmission and electroluminescence (EL) measurements were obtained using a Bruker Vertex 70 Fourier transform infrared (FTIR) spectrometer. The system is associated with a temperature-controlled Oxford Instruments continuous flow helium cryostat to record temperature-dependent EL spectra. A molecular sieve drying column was used to filter out atmospheric $\mathrm{CO}_{2}$ and $\mathrm{H}_{2} \mathrm{O}$ from the optical path.

In order to realize a practical RCLED, it is necessary to calculate the emission rate enhancement at the resonance wavelength, $G_{e}$ and the integrated emission enhancement, $G_{\text {int }}$ which can be derived as: ${ }^{31}$

$$
\begin{aligned}
& G_{e} \approx \frac{\xi}{2} \frac{2}{\pi} \frac{\pi\left(R_{1} R_{2}\right)^{1 / 4}\left(1-R_{1}\right)}{\left(1-\sqrt{R_{1} R_{2}}\right)^{2}} \frac{\tau_{c a v}}{\tau} \\
& G_{\text {int }} \approx G_{e} \sqrt{\pi \ln 2} \frac{\Delta \lambda_{\text {cav }}}{\Delta \lambda_{\text {ref }}}
\end{aligned}
$$

where $R_{1}$ and $R_{2}$ are the reflectivity of the top and bottom DBR mirror, respectively, $\xi$ is the antinode enhancement factor, and $\Delta \lambda_{r e f}$ is the linewidth of the active region. $\tau_{c a v}$ and $\tau$ are the lifetime of the cavity and the active region, respectively. The linewidth of the resonant cavity mode $\Delta \lambda_{\text {cav }}$ is related to the cavity quality factor $Q$ via the relation: ${ }^{31}$

$$
\Delta \lambda_{\text {cav }}=\frac{\lambda_{\text {cav }}}{Q}=\frac{\lambda_{c a v}}{m} \frac{1-\sqrt{R_{1} R_{2}}}{\pi \sqrt[4]{R_{1} R_{2}}}
$$

where $\lambda_{\text {cav }}$ is the position of the mode. The cavity order $m$ is related to the cavity thickness $d_{\text {cav }}$ by $m=2 n_{\text {cav }} d_{\text {cav }} / \lambda_{\text {cav }}$, where $n_{\text {cav }}$ is the refractive index of the cavity. For low values of $m$, high extraction efficiency should be achieved owing to the greater overlap between the cavity response and the underlying emission spectrum. ${ }^{24,32}$ Therefore, we selected $d_{\text {cav }}=\lambda$ corresponding to $m=2$. 
Figures 2(a) and 2(b) show the emission rate enhancement at the resonance wavelength and the integrated emission enhancement as a function of the DBR mirror reflectivity. The highest integrated emission enhancement $\left(G_{\text {int }}\right)$ is achieved when $R_{2}>95 \%$, and $50 \%<R_{1}<R_{2}$. Furthermore, in order to obtain a high emission enhancement factor $\left(G_{e}\right)$ of more than 30 times, $R_{2}$ and $R_{1}$ should be greater than $96 \%$ and $82 \%$, respectively. The reflectivity of the DBR mirrors was modelled by a transfer matrix method ${ }^{33}$, using the refractive indices of $n_{L} \approx 3.16$ for AlAsSb and $n_{H} \approx 3.76$ for GaSb. The main advantage of this layer combination is the large refractive index contrast $(\Delta n \sim 0.6)$, which requires fabricating only a few quarter- $\lambda$-thick layer pairs to achieve high reflectivity. The simulation results indicate that 13.5 periods of the bottom DBR and 5 periods of the top DBR are sufficient to achieve high reflectivity, $R_{2}>97 \%$ and $R_{1}>83 \%$, as shown in Fig. 2(c). The optical transmittance spectrum of the full RCLED structure measured at room temperature is shown in Fig. 2(d). The peak of the mean resonant optical mode cavity at $4.295 \mu \mathrm{m}$ is slightly detuned from the center of the DBR stopband at $4.180 \mu \mathrm{m}$. The detuning, which is given by the difference between $\lambda_{\text {cav }}$ and $\lambda_{D B R}$ is considered in Fig. 2(e) and 2(f). It was found that the values of $G_{\text {int }}$ and $G_{e}$ fall within the high range - i.e. no less than $6 \%$ and $15 \%$ of the maximum value compared to the resonance case, respectively.

The electroluminescence (EL) emission spectra of the InAsSb RCLED and the reference LED are shown in Fig. 3(a) and 3(b), respectively. Over the measured temperature range (20$300 \mathrm{~K}$ ) the main peak intensity of the RCLED and the EL emission peak of the reference LED decreased with increasing temperature by a factor of $\sim 68$ and $\sim 32$, respectively. The EL emission peak of the RCLED also shifts to longer wavelengths with increasing temperature but much more slowly compared to the reference LED. In the conventional LED the wavelength shift of the EL spectrum is due to the temperature dependence of the active region bandgap, 
whereas, for the RCLED, the EL peak depends on the wavelength of the resonant cavity optical mode which is determined by the thickness and the refractive index of the materials forming the cavity. With an increase in temperature, the thickness of the cavity increases according to the thermal expansion coefficients of the cavity materials. We calculated the variation of the cavity thickness as $\sim 1.6 \mathrm{~nm}$. This represents $<4 \%$ of the total wavelength shift and therefore the main contribution comes from the temperature variation of the refractive index. ${ }^{34,35}$ Consequently, the main EL emission peak of the RCLED shifts only by $\sim 100 \mathrm{~nm}$ at a rate of $0.35 \mathrm{~nm} / \mathrm{K}$, compared with $\sim 600 \mathrm{~nm}$ at a rate of $\sim 2.15 \mathrm{~nm} / \mathrm{K}$ in the reference LED (see Fig. 3(c)). This represents more than a factor of 6 improvement in the emission wavelength stability with temperature, which is useful in applications such as gas detection where wavelength stability is required to ensure the emission peak remains within the gas absorption envelope as the temperature varies. The far-field intensity profile of the RCLED at $300 \mathrm{~K}$ is shown in Fig. 3(c) inset. A single lobe intensity distribution centred at $0^{\circ}$ with a half angle of $60^{\circ}$ was obtained, in good agreement with previous work on RCLEDs at shorter wavelengths ${ }^{36}$.

The full width at half maximum (FWHM) of the RCLED emission spectra are clearly narrower than those of the conventional LED. For the RCLED the FWHM of the EL emission increased from $35 \mathrm{~nm}$ at $20 \mathrm{~K}$ to $88 \mathrm{~nm}$ at $300 \mathrm{~K}$, whereas the reference LED had a linewidth of $250 \mathrm{~nm}$ at $20 \mathrm{~K}$ and $900 \mathrm{~nm}$ at $300 \mathrm{~K}$. The linewidth of the reference LED is determined by the joint density of states and the thermal energy of carriers - typically FWHM $1.8 \mathrm{kT}^{21}$ In contrast, for the RCLED, the linewidth depends on the quality factor of the resonant cavity. At room temperature, the emission spectrum of the RCLED has a linewidth $\sim 10 \mathrm{x}$ narrower than that of the conventional reference LED. Consequently, the RCLED can be designed and tailored to match the absorption wavelength of the target gas.

In order to evaluate the emission enhancement factor, the measured EL spectrum of the RCLED and the reference LED spectrum have been plotted together at $300 \mathrm{~K}$, as shown in 
Fig. 3(d). A strong emission enhancement at the resonant wavelength is obtained in close agreement with our resonant cavity design, and in particular due to the reflectivity of the DBR mirrors and the positioning of the active region at the electric field antinode within the cavity $(\xi=2)$. The resonant peak emission and the integrated peak emission are enhanced by a factor of $\sim 70$ and $\sim 11$, respectively. The total integrated emission enhancement was calculated to be around 33 times. Due to more reflective DBR mirrors and higher cavity $Q$-factor, our results show greater enhancement values (x10) compared with previous work ${ }^{24,30}$. The output power of the RCLED was measured to be $5.5 \mu \mathrm{W}$ (external efficiency $\approx 0.024 \%$ ) at $300 \mathrm{~K}$ rising to $45 \mu \mathrm{W}$ at $77 \mathrm{~K}$. We note that the electroluminescence of our RCLED lies entirely within the $\mathrm{CO} 2$ absorption band which coupled with the improved directionality provides a higher level of useful emission intensity compared with previous work $3,5,13-18$.

In conclusion, we have demonstrated a mid-infrared RCLED operating near $4.2 \mu \mathrm{m}$ at room temperature, grown on a GaSb substrate by molecular beam epitaxy. The RCLED structure consists of a $1 \lambda$-thick microcavity sandwiched between two high contrast DBR mirrors. Due to the resonant cavity effects, the resonance peak emission and the integrated peak emission were found to be enhanced by a factor of $\sim 70$ and $\sim 11$, respectively, while the total integrated emission enhancement was $\sim \mathrm{x} 33$. The cavity mode of the RCLED was observed to shift with temperature at a rate of only $0.35 \mathrm{~nm} / \mathrm{K}$, which is less than that of the reference LED by a factor of 6 . Also, at room temperature, the linewidth of the RCLED emission spectrum is x10 narrower than that of the reference LED. Owing to the increased brightness, narrower linewidth and improved temperature dependence our results indicate that this RCLED design forms an excellent basis for the further development of sources to be used in gas sensor instruments for the detection of $\mathrm{CO}_{2}$ as well in spectroscopy and other applications. 


\section{ACKNOWLEDGEMENTS}

Furat A. Al-Samari has been supported by a studentship funded by the ministry of higher education in Iraq.

\section{FIGURE CAPTIONS}

Fig. 1. (a) Schematic diagram of the InAsSb resonant cavity structure. (b) SEM cross-section of the RCLED sample (marker represents $1 \mu \mathrm{m}$ ). (c) Schematic of the completed RCLED.

Fig. 2. Room temperature simulation results of (a) the resonant emission $\left(G_{e}\right)$ and (b) the integrated emission $\left(G_{i n t}\right)$ enhancement factor as a function of the top and bottom DBR mirror reflectivity, $R_{1}$ and $R_{2}$, respectively. (c) Simulation results of the DBR reflectivity as a function of the wavelength for 13.5 pairs (bottom DBR) and 5 pairs (top DBR). (d) Experimentally measured transmittance spectrum of the RCLED structure. Room temperature simulations of (e) $G_{e}$ and (f) $G_{i n t}$ as a function of the DBR stopband center and the peak emission of the cavity mode, respectively. The experimentally determined values of the stopband centre and the position of the cavity mode are shown as open square points. The white dashed lines in the color-bar represent the values of $G_{e}$ and $G_{i n t}$.

Fig. 3. Temperature dependence of the EL spectra for (a) the RCLED and (b) the reference LED, showing the wavelength of the main EL emission peak at room temperature occurs at $4.295 \mu \mathrm{m}$, which corresponds to the cavity resonance wavelength shown in Fig. 2(d). (c) The position of the EL peak as a function of the temperature. The inset is the far-field angular profile of the RCLED at $300 \mathrm{~K}$, (d) EL emission spectra for the RCLED (solid curve) and reference LED (dashed curve). 


\section{REFERENCES}

${ }^{1}$ A. Krier, H. H. Gao, V. V. Sherstnev, Y. Yakovlev, J. of Phys. D: Appl. Phys. 32, 3117 (1999).

${ }^{2}$ H. H. Gao, A. Krier, V. Sherstnev, Y. Yakovlev, J. of Phys. D: Appl. Phys. 32, 1768 (1999).

${ }^{3}$ M. J. Pullin, H. R. Hardaway, J. D. Heber, C. C. Phillips, W. T. Yuen, Ra A. Stradling, P. Moeck, Appl. Phys. Lett. 74, 2384 (1999).

${ }^{4}$ A. Krier, V. V. Sherstnev, J. of Phys. D: Appl. Phys. 33, 101 (2000).

${ }^{5}$ P. J. Carrington, Q. Zhuang, M. Yin, A. Krier, Semi. Sci. and Tech. 24, 075001 (2009).

${ }^{6}$ D. Lackner, M. Steger, M. L. W. Thewalt, O. J. Pitts, Y. T. Cherng, S. P. Watkins, E. Plis, S. Krishna, J. of Appl. Phys. 111, 034507 (2012).

${ }^{7}$ A. P. Craig, A. R. J. Marshall, Z-B. Tian, S. Krishna, A. Krier, Appl. Phys. Lett. 103, 253502 (2013).

${ }^{8}$ M. Aziz,; Ch. Xie, V. Pusino, A. Khalid, M. Steer, I.G. Thayne, D. RS. Cumming, Appl. Phys. Lett. 111, 102102 (2017).

${ }^{9}$ L. Meriggi, M. J. Steer, Y.Ding, I.G. Thayne, C. MacGregor, Ch. N. Ironside, M. Sorel, J. of Appl. Phys. 117, 063101 (2015).

${ }^{10}$ H. Aït-Kaci, J. Nieto, J. B. Rodriguez, P. Grech, F. Chevrier, A. Salesse, A. Joullié, P. Christol, Physica status solidi (a) 202, 647-651 (2005).

${ }^{11}$ A. Krier, M. Stone, S. E. Krier, Semi. Sci. and tech. 22, 624 (2007).

${ }^{12}$ A. P. Craig, M. D. Thompson, Z. B. Tian, S. Krishna, A. Krier, A. R. J. Marshall, Semi. Sci. and Tech. 30, 105011 (2015).

${ }^{13}$ A. S. Golovin, A. P. Astakhova, S. S. Kizhaev, N. D. Il'inskaya, O. Yu. Serebrennikova, Yu. P. Yakovlev, Technical Physics Letters 36, 47 (2010). 
${ }^{14}$ M. K. Haigha, G. R. Nash, S. J. Smith, L. Buckle, M. T. Emeny, T. Ashley, Appl. Phys. Lett. 90, 231116 (2007).

${ }^{15}$ N. V. Zotova, N. D. Il'inskaya, S. A. Karandashev, B. A. Matveev, M. A. Remennyi, M. N. Stus', Semiconductors 40, 697 (2006).

${ }^{16}$ P. J. Carrington, V. A. Solov'ev, Q. Zhuang, A. Krier, S. V. Ivanov, Appl. Phys. Lett. 93, $091101(2008)$.

${ }^{17}$ A. Krier, M. Stone, Q. D. Zhuang, Po-Wei Liu, G. Tsai, H. H. Lin, Appl. Phys. Lett. 89, 091110 (2006).

${ }^{18}$ J.A. Keen, E. Repiso, Q. Lu, M. Kesaria, A.R.J. Marshall, A. Krier, Infrared Physics \& Technology 93, 375 (2018).

${ }^{19}$ C. Wiesmann, K. Bergen ek, N. Linder, U. T. Schwarz, Laser \& Photon. Rev. 3, 262 (2009).

${ }^{20}$ A. I. Zhmakin, Physics report 498, 189 (2011).

${ }^{21}$ E. F. Schubert, Y-H. Wang, A. Y. Cho, L-W. Tu, G. J. Zydzik, Appl. Phys. Lett. 60, 921 (1992).

${ }^{22}$ N. E. J. Hunt, E. F. Schubert, R. A. Logan, G. J. Zydzik, Appl. Phys. Lett. 61, 2287 (1992).

${ }^{23}$ E. Hadji, J. Bleuse, N. Magnea, J. L. Pautrat, Appl. Phys. Let. 67, 2591-2593 (1998).

${ }^{24}$ A. M. Green, D. G. Gevaux, Ch. Roberts, Ch. C. Phillips, Physica E: Low-dimensional Systems and Nanostructures 20, 531 (2004).

${ }^{25}$ A. Ducanchez, L. Cerutti A. Gassenq, P. Grech, F. Genty, IEEE J. of Sel. Top. in Quant. Electro. 14, 1014 (2008). 
${ }^{26}$ Ch. Grasse, P. Wiecha, T. Gruendl, S. Sprengel, R. Meyer, M-C. Amann, Appl. Phys. Lett. 101, 221107 (2012).

${ }^{27}$ A. Bachmann, Sh. Arafin, K. Kashani-Shirazi, New J. of Phys. 11, 125014 (2009).

${ }^{28}$ A. Andrejew, S. Sprengel, M-C. Amann, Opt. Lett. 41, 2799 (2016).

${ }^{29}$ G. K. Veerabathran, S. Sprengel, A. Andrejew, M-C.Amann, Appl. Phys. Lett. 110, 071104 (2017).

${ }^{30}$ L. Meriggi, M. J. Steer, Y. Ding, I. G. Thayne, C. MacGregor, Ch. N. Ironside, M. Sorel, IEE $11^{\text {th }}$ Conference on Ph.D. Research in Microelectronics and Electronics (PRIME) 180 (2015).

${ }^{31}$ E. Fred Schubert, Light Emitting Diodes, Cambridge Press (2006).

${ }^{32}$ H. Benisty, H. De Neve, C. Weisbuch, IEEE J. of Quant. Electron. 34, 1612 (1998).

${ }^{33}$ M. Born, E. Wolf, Principles of optics, Cambridge University Press (1999).

${ }^{34}$ W. W. Bewley, C. L. Canedy, C. S. Kim, C. D. Merritt, M. V. Warren, I. Vurgaftman, J. R. Meyer, M. Kim, Appl. Phys. Lett. 109, 151108 (2016).

${ }^{35}$ A. B. Ikyo, I. P. Marko, K. Hild, A. R. Adams, S. Arafin, M-C. Amann, S. J. Sweeney, Sci. Rep. 6, 19595 (2016).

${ }^{36}$ R. H. Birkner, j. Kaiser, W. ElsÄßer, C. Jung, Appl. Phys. B 79, 963-967 (2004). 
(a)
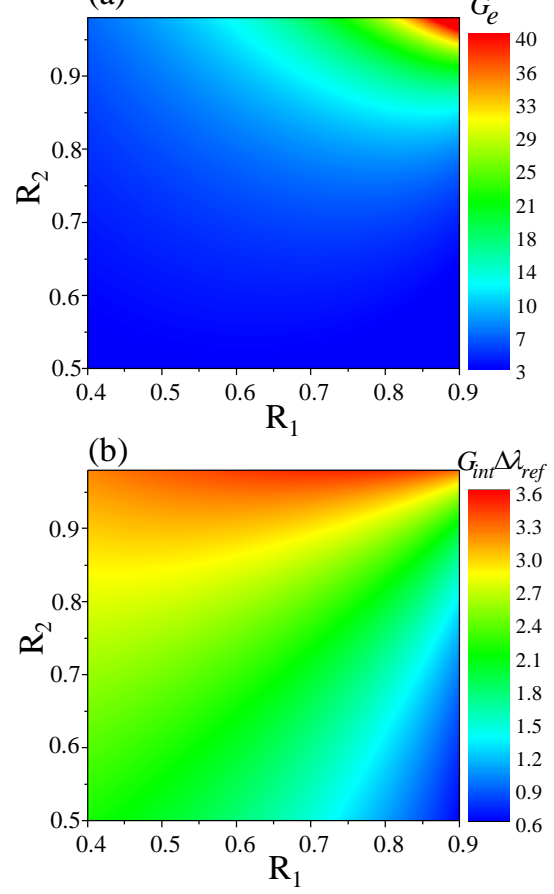
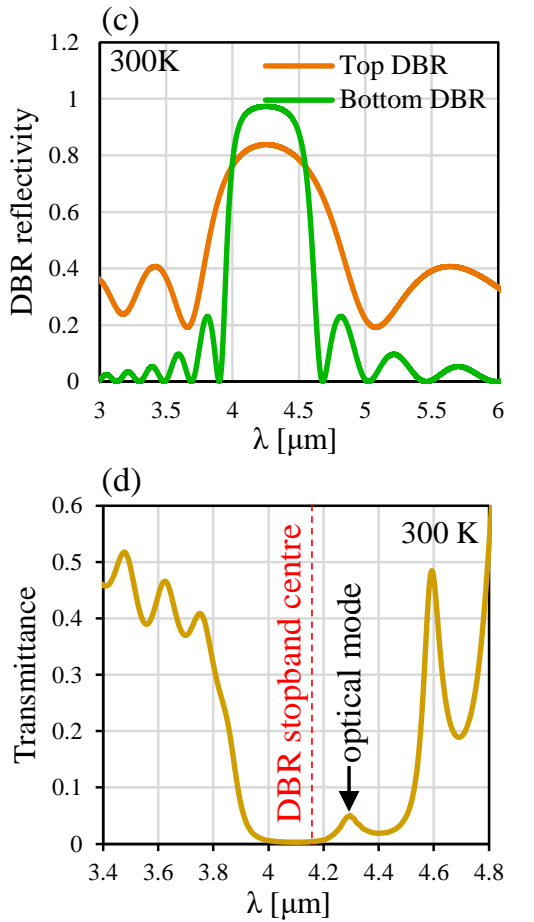
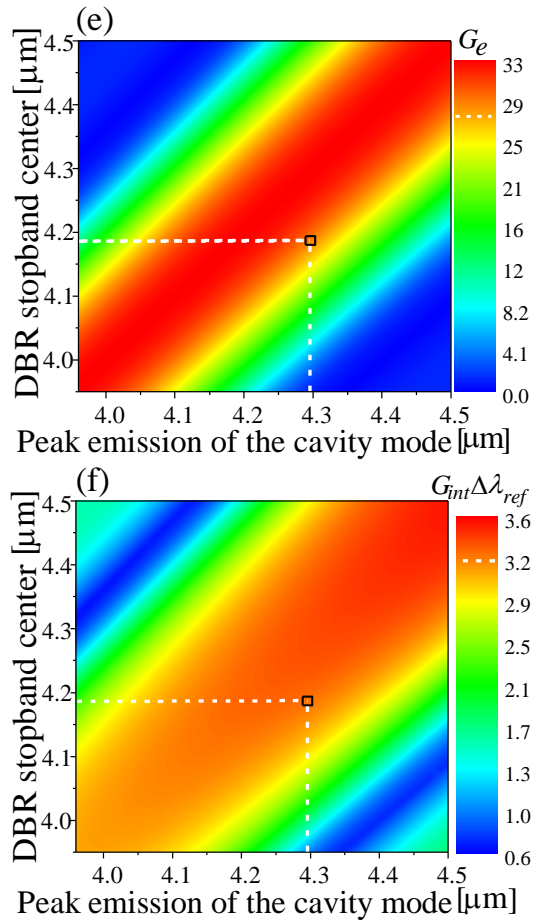
